\title{
Estudio comparativo macrofauna del suelo en sistema agroforestal, potrero tradicional y bosque latifoliado en microcuenca del trópico seco, Tomabú, Nicaragua
}

\author{
Alexa del Carmen Escobar Montenegro ${ }^{1}$ \\ Jordi Bartolomé Filella² \\ Noel Antonio González Valdivia ${ }^{3}$
}

\section{RESUMEN}

Perturbaciones ocasionadas por cambios de usos, como la conversión del bosque a pastos, podrían modificar la composición de la edafofauna y afectar la probabilidad de recuperación del ecosistema original, en Nicaragua existen pocas investigaciones acerca de los efectos de este cambio de uso de suelos sobre las comunidades edáficas. El objetivo del presente estudio fue identificar y comparar la diversidad de la macro fauna de suelo en tres sistemas de uso del suelo: sistema silvopastoril, potrero tradicional y bosque latifoliado en una microcuenca del trópico seco centroamericano. También se valoró la relación de la macro fauna con las características fisicoquímicas del suelo mediante análisis estadísticos de regresiones lineales. El estudio se realizó en la microcuenca Tomabú de 3,676.6 ha en el norte de Nicaragua, Centroamérica. La extracción de macro fauna del suelo se realizó mediante el sistema de Berlesse-Tüllgreen y el uso de trampas pitfall o trampas de caída. En el análisis fisicoquímico del suelo se determinó textura, $\mathrm{pH}$, materia orgánica, capacidad de intercambio catiónico, nitrógeno, fósforo, potasio, calcio, magnesio, hierro, cobre, zinc, manganeso. La diversidad se determinó mediante el cálculo del índice de Shannon. Los resultados mostraron que la riqueza taxonómica encontrada fue 27 grupos de artrópodos identificados a nivel de orden, con 9,880 individuos recolectados, entre los que se destacan los órdenes de himenópteras, ácaros e isópteras. También, los suelos presentaron características con valores numéricos similares en $\mathrm{pH}$, materia orgánica, nitrógeno, fósforo y capacidad de intercambio catiónico. Se puede concluir que en la microcuenca de Tomabú existe una mayor diversidad de macro fauna del suelo en el sistema de bosque comparada con los sistemas de producción pecuaria del estudio, aunque no se presentan diferencias estadísticas significativas, al aplicar los análisis de ANDEVA, lo cual se asocia al $\mathrm{pH}$ del suelo y la materia orgánica.

Palabras clave: Edafofauna, biodiversidad, Berlesse, Pitfall, macro invertebrados, Nicaragua.

Recibido: 07 de junio de 2017

Aceptado: 26 de junio de 2017

1 Universidad Nacional Autónoma de Nicaragua, Facultad Regional Multidisciplinaria de Estelí. Estelí (Nicaragua). Correo electrónico: ratmazy@gmail.com

2 Universidad Autónoma de Barcelona. Facultad de Veterinaria. Correo electrónico: Jordi.bartolome@uab.cat

3 El Colegio de la Frontera Sur, Tabasco, México. siankaan2003@gmail.com 


\title{
A comparative study of soil macrofauna in agroforestry systems, traditional broadleaf forest and pasture in the dry tropical watershed, Tomabu; Nicaragua
}

\begin{abstract}
Disturbances caused by different changes such as the conversion from forest to pastures could modify the composition of the soil fauna and affect the probability of recovery of the original ecosystem. In Nicaragua there are few studies on the effects of this change of soil use on the edaphic communities. This study aims to identify and compare the soil macro fauna diversity in three systems of soil use: silvopastoral system, traditional pasture and broadleaf forest in a micro-watersheds of Central America dry tropics. The relation between macrofauna with the physicochemical characteristics of the soil conducted by statistical analysis of linear regressions. The study was carried out in the watershed Tomabú, which covers 3,676 hectares in the north of Nicaragua, Central America. The extraction of macrofauna of the soil as was obtained by the system of Berlesse-Tüllgreen and the use of pitfall. In the physicochemical analysis from soil, texture, organic matter, cation exchange capacity, nitrogen, phosphorus, potassium, calcium, magnesium, iron, copper, zinc, manganese, were determined. Diversity was determined by calculating the Shannon index. The results showed that the taxonomic richness found were 27 groups of arthropods identified at the level of order, with 9,880 collected individuals, among which hymenoptera, mites and isopteran are stand out. In the same way, the soils showed characteristics with similar numerical values in $\mathrm{pH}$, organic matter, nitrogen, phosphorus and cation exchange capacity. It can be concluded that in Tomabú watershed there is a greatest diversity of soil macrofauna of forest system compare to the systems of livestock production of the study. However, there are no significant statistical differences when applying the analysis of ANDEVA, which is associated with soil $\mathrm{pH}$ and organic matter.
\end{abstract}

Keywords: Soil fauna, biodiversity, pitfall, macro invertebrates, Nicaragua 


\section{INTRODUCCIÓN}

El sistema suelo es el resultado de complejas interacciones entre factores físicos, químicos $\mathrm{y}$ biológicos, (Castro, 2007) y las comunidades edáficas se consideran las más ricas en especies de todos los ecosistemas terrestres (Anderson, 1975; Ghilarov, 1977; Stanton, 1979). Los macro invertebrados edáficos $(>2$ mm diámetro) actúan como agentes determinantes en la fertilidad del suelo y, por ende, en el funcionamiento global del sistema edáfico(Cabrera et al., 2011), también conocidos como macro fauna; tienen diferentes efectos sobre los procesos que determinan el crecimiento de las plantas (Lavelle, 1995). En los sistemas tropicales los macro invertebrados desempeñan una función clave en los procesos que determinan la conservación y fertilidad del suelo, al regular la disponibilidad de minerales asimilables por las plantas y favorecer la estructura del suelo, influyendo en las condiciones de vida, la abundancia y composición de las comunidades del suelo o edáficas (Feijoo y Knapp, 1998).

Sin embargo, prácticas antrópicas alteran la composición de las comunidades edáficas (Arroyo et al., 2003), ya que la pérdida de biodiversidad asociada a la transformación de ecosistemas naturales a cultivo modifica el flujo de nutrientes y energía que requieren la intervención humana para mantener la función productiva del sistema (Altieri, 1999). Las perturbaciones ocasionadas por cambios de usos, como la conversión del bosque a pastos o a sistemas silvopastoriles podrían modificar la composición de la edafofauna y afectar la probabilidad de recuperación del ecosistema original.

En la microcuenca Tomabú, donde hay un área protegida (MARENA-CARE-PIMCHAS, 2008) al menos $80 \%$ del territorio se usa para la ganadería y en $47.6 \%$ (61 fincas) hay sistemas silvopastoriles, pero en Nicaragua hay pocas investigaciones acerca de los efectos de este cambio de uso de suelos sobre las comunidades edáficas. La hipótesis de este estudio fue que la biodiversidad de macro fauna del suelo es mayor en el bosque que en los sistemas de producción pecuaria. El objetivo fue identificar y comparar la diversidad de macro fauna presente en el suelo de un sistema silvopastoril, un potrero tradicional y un bosque latifoliado del trópico seco, considerando las condiciones fisicoquímicas de estos suelos.

\section{MATERIALES Y MÉTODOS}

\section{Área de estudio}

La microcuenca Tomabú se encuentra en La Trinidad, municipio del departamento de Estelí, al norte de Nicaragua, con una extensión territorial de 3,676.6 ha. El clima es tropical seco (INIFOM, 2012), la precipitación anual varía de 800 a 1,200 mm, con una humedad relativa de $63 \%$ y temperatura media anual entre 20 a $22{ }^{\circ} \mathrm{C}$; la época seca dura 5 a 7 meses. Las altitudes máxima y mínima son 674 y 1,444 msnm (CARE-MARENA-PIMCHAS, 2008)

Los suelos son de color pardo grisáceo, con textura arcillosa y franco arcillosa; $70 \%$ de la topografía es ondulada con pendientes pronunciadas, porcentajes altos de pedregosidad, y, en muchos casos, erosionados (INIFOM, 2012).

\section{Selección de la muestra y muestreo}

Al menos $80 \%$ de este territorio se usa para la ganadería y resulta un suelo sobre utilizado (MARENA, CARE, PIMCHAS, 2008). Del universo de fincas con sistemas silvopastoriles (SSP) en la microcuenca (61fincas) se extrajo una muestra de $5 \%$, mediante un muestreo estratificado utilizando la fórmula del factor de muestreo $\mathrm{n} / \mathrm{N}$ (donde $\mathrm{n}$ es el tamaño de la muestra $\mathrm{y}$ $\mathrm{N}$ es el tamaño de la población) (Piura, 1994), esta muestra se comparó con su equivalente de fincas con sistema de potrero tradicional y sistema de bosque latifoliado, distribuidos en la micro cuenca. 
Los SSP de la zona encajan con la descripción de Mahecha (2002) se consideran como una opción de producción pecuaria donde las leñosas perennes (árboles o arbustos) interactúan con los componentes tradicionales (forrajeras herbáceas y animales) en un sistema de manejo integral. En este estudio, la distribución espacial de los sistemas silvopastoriles fue de árboles dispersos de regeneración natural Guazuma ulmifolia, Acacia pennatula (Casasola et al., 2001), Psiduim guajava, cobertura de gramíneas: Andropogon gayanus, y cercas vivas con leguminosas Gliricidia Sepium y Caesalpinia velutina (MARENA / INAFOR, 2002).

El sistema de potrero tradicional (SPT), está compuesto por áreas de pastos en monocultivos, de Paspalum notatum y Andropogon gayanus, donde también aparecen algunas malezas arbustivas.

El sistema de bosque (SB), es del tipo tropical seco latifoliado, que en Nicaragua abarca desde bosques densos hasta ralos, con gran proporción de especies xerófitas, perdiendo el follaje en la época seca. Presentan uno o dos estratos y son relativamente pobres en su composición florística (MARENA / INAFOR, 2002).

Se delimitaron parcelas de 1 ha en cada sistema con tres replicas por sistema, teniendo un total de nueve parcelas. Para realizar el muestreo biológico, en cada parcela se definieron cinco puntos de muestreo, de donde se sustrajeron las muestras de suelo con ayuda de una sonda extractora metálica con medidas de: $7 \times 7 \mathrm{~cm}$ x20 cm de profundidad. Para la sustracción de las muestras del suelo, en el terreno se trazaron dos círculos concéntricos a 3 y $6 \mathrm{~m}$ de radio, respectivamente; se colocaron cinco trampas de caída (pitfall), para complementar el muestreo, localizadas a $14 \mathrm{~m}$ del centro, siguiendo el procedimiento de Moreira et al., (2012). El traslado de las muestras se hizo el mismo día de su recolección, en sacos de tela gruesa de color oscuro, para evitar el deterioro de los especímenes, hasta el laboratorio de la Estación experimental del trópico seco El Limón UNAN FAREM Estelí, donde se hizo la extracción de la macrofauna, identificación y clasificación.

Se obtuvo un total de 45 muestras de suelo a 20 $\mathrm{cm}$ de profundidad, las cuales fueron montadas durante siete días usando una versión modificada del sistema Berlesse-Tullgren como lo describe Peredo, (2012) para la extracción de macrofauna. También se obtuvieron 45 muestras biológicas de las trampas pitfall. En la extracción de macrofauna con el sistema Berlesse se utilizaron focos de 40watts.

El muestreo de macrofauna se realizó con sonda extractora metálica, para no alterar la estructura del suelo (Domínguez et al., 2001). Los muestreos se efectuaron al final de la época de lluvias (Noviembre 2012) que, según Huerta et al., (2008) es cuando existe mayor actividad de la macrofauna.

La macrofauna extraída se conservó en alcohol de $70^{\circ}$ (Arroyo et al., 2003; Zerbino et al., 2008). Los especímenes obtenidos fueron estudiados con un microscopio estéreo y contados e identificados taxonómicamente hasta nivel de orden (Zerbino, 2010; Peredo et al., 2012).

Para el análisis físico-químico de suelo se tomó una muestra homogénea representativa de cada parcela. Para la obtención de estas muestras se aplicó un patrón de muestreo en diagonal y se obtuvieron 20 sub muestras de las cuales se extrajo una muestra usando el método del cuarteo como lo describen Aloé et al., (2007), para garantizar la representatividad. La profundidad de extracción fue a $15 \mathrm{~cm}$, según los requerimientos del laboratorio de Suelos y aguas que describe UNA, (2012) donde se realizaron los análisis. La estructura se determinó en campo.

Las variables de suelo que se tomaron en cuenta en el estudio, fueron: $\mathrm{pH}$, materia orgánica (MO), 
capacidad de intercambio catiónico (CIC), textura, macro elementos: Nitrógeno $(\mathrm{N})$, Fosforo $(\mathrm{P})$, Potasio $(\mathrm{K})$, Calcio $(\mathrm{Ca})$, Magnesio $(\mathrm{Mg})$ y micro elementos: Hierro (Fe), Cobre (Cu), Zinc ( $\mathrm{Zn})$, Manganeso (Mn).

\section{Procesamiento estadístico de datos}

Con los datos de biodiversidad de la macrofauna se calculó el Índice de diversidad de Shannon (H' $=\Sigma$ pilog2pi), que expresa la uniformidad de los valores de importancia a través de todas las especies de la muestra; este asume que los individuos son seleccionados al azar y que todas las especies están representadas en la muestra (Magurran, 1988). La densidad de individuos se obtuvo a partir del número de individuos por unidad muestreada.

Para comparar los resultados de biodiversidad y abundancia en los tres sistemas se aplicó un ANDEVA para datos paramétricos cuando los datos cumplían con los principios de normalidad y una comparación de medias mediante el criterio de diferencia mínima significativa con Tukey $(\mathrm{P} \leq 0.05)$. Así mismo se realizaron regresiones lineales para determinar la relación entre características del suelo con la diversidad y abundancia encontrada en el estudio. Para determinar la normalidad de los datos de diversidad se realizó la prueba de Kolmogorov-Smirnov para una muestra, con el programa estadístico Stat Wiew.

\section{RESULTADOS Y DISCUSIÓN}

\section{Abundancia y diversidad de macrofauna edáfica}

La riqueza taxonómica, encontrada fue de 27 grupos de artrópodos identific entificados y 8425 individuos seguido por la clase arácnida con 5 órdenes y 1411 individuos de los cuales los ácaros ocupan el 69\%.

Cuadro 1. Riqueza taxonómica ( $\mathrm{n}^{\circ}$ individuos) de la macro fauna recolectada para los tres sistemas en estudio: sistema silvopastoril (SSP), sistema bosque latifoliado (SB) y sistema de potrero tradicional (SPT) de la micro cuenca Tomabú, Nicaragua. 2012.

\begin{tabular}{|c|c|c|c|c|c|c|}
\hline Clase & Sistema Orden & SSP & & PT & Total & Abundancia/clase \\
\hline Oligoqueta & Oligoqueta & 4 & 0 & 0 & 4 & 4 \\
\hline Gasterópoda & Moluscos & 4 & 20 & 0 & 24 & 24 \\
\hline Miriópoda & Quilópodos & 9 & 4 & 3 & 16 & 16 \\
\hline \multirow[t]{5}{*}{ Arácnida } & Ácaros & 259 & 449 & 268 & 976 & 1411 \\
\hline & Araneidos & 106 & 100 & 79 & 285 & \\
\hline & Amblypygi & 5 & 0 & 2 & 7 & \\
\hline & Scorpion & 0 & 1 & 2 & 3 & \\
\hline & Opillion & 56 & 26 & 58 & 140 & \\
\hline \multirow[t]{9}{*}{ Insecta } & Colémbolos & 308 & 203 & 189 & 700 & 8425 \\
\hline & Díptera & 110 & 166 & 89 & 365 & \\
\hline & Himenóptera & 2190 & 1056 & 1264 & 4510 & \\
\hline & Lepidóptera & 89 & 77 & 60 & 226 & \\
\hline & Coleóptera & 291 & 307 & 163 & 761 & \\
\hline & Homóptera & 250 & 131 & 146 & 527 & \\
\hline & Psocóptera & 2 & 0 & 1 & 3 & \\
\hline & Dermáptera & 81 & 45 & 28 & 154 & \\
\hline & Macoptera & 0 & 9 & 0 & 9 & \\
\hline
\end{tabular}




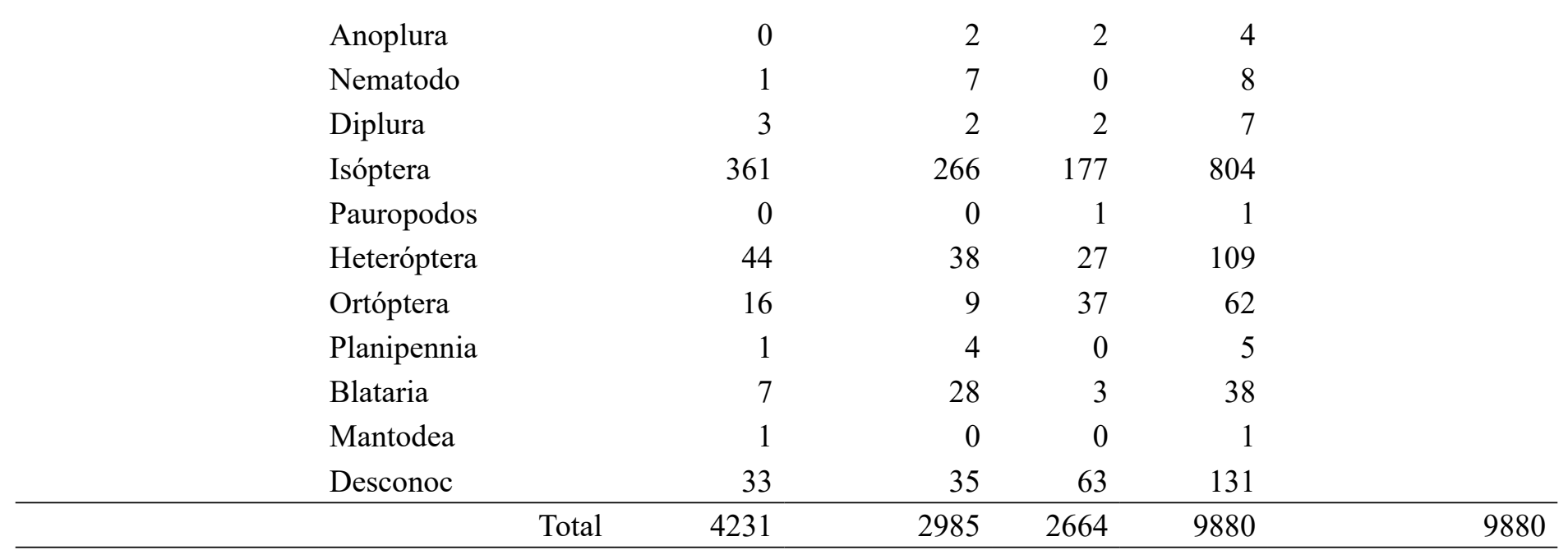

En la clase insecta se destaca el orden himenóptera, que diversos autores las ubican dentro de la macrofauna edáfica, como uno de los grupos más abundantes y diversos, que desempeñan una función muy importante como depredadoras, herbívoras o detritívoras, y participan en los procesos físico químicos del suelo (Lavelle y Kohlman, 1984; Delabie y Fowler,1990; Fernández, 2001). También resalta el orden Coleóptera, que Zerbino (2010), identifica como grupos taxonómicos que mostraron ser más sensibles a los cambios en el uso de suelos y que en este caso tienen mayor abundancia en el bosque latifoliado seguido del sistema silvopastoril y por último en el sistema de potrero tradicional, lo cual podría tomarse como un indicativo del grado de perturbación del suelo.

Dentro de la clase arácnida se destaca el orden de los ácaros, que han sido estudiados por su particular abundancia en el suelo y la diversidad de funciones en las que se involucran (Gil, 1997; Arroyo et al., 2003; Socarras y Robaina, 2011; Rodríguez, 2012) también han sido objeto de estudio como indicadores de calidad de suelos ya que algunas de sus familias son sensibles a los cambios de uso de suelo mientras otras predominan en suelos disturbados.

En este estudio la mayor cantidad de individuos fue registrada en el bosque latifoliado, seguido del sistema de potrero tradicional, y sería idóneo continuar esta investigación hasta identificar que familias son las que predominan para poder relacionar su presencia con las funciones específicas que tienen en el suelo.

Después de las dos clases más representativas en este estudio, se encuentran los gasterópodos donde los especímenes encontrados en primer lugar fueron en el bosque latifoliado, en segundo lugar en el sistema silvopastoril y en el potrero tradicional no se encontró ninguno, lo que llama la atención de acuerdo a lo planteado por González et al, (2011) quienes proponen los gasterópodos como indicadores de biodiversidad para bosques del trópico con algunas especies muy sensibles a la perturbación.

Como se presenta en la Figura 1, existen diferencias significativas entre la abundancia de individuos del sistema silvopastoril y los sistemas de bosque y potrero tradicional. Numéricamente el sistema silvopastoril registró la mayor abundancia de individuos de macrofauna y mayor número de grupos taxonómicos hasta el nivel de orden, predominando el orden himenóptera seguido por isóptera, colémbolos, coleópteras y ácaros. Seguido en abundancia estuvo el sistema bosque latifoliado con predominancia igualmente de himenópteras, pero seguido de los ácaros, coleópteras, isóptera y colémbolos. En el sistema de potrero tradicional se registra la menor abundancia de individuos y de grupos taxonómicos donde después de himenópteras se encontraron los ácaros, colémbolos, isópteras y coleópteras. 


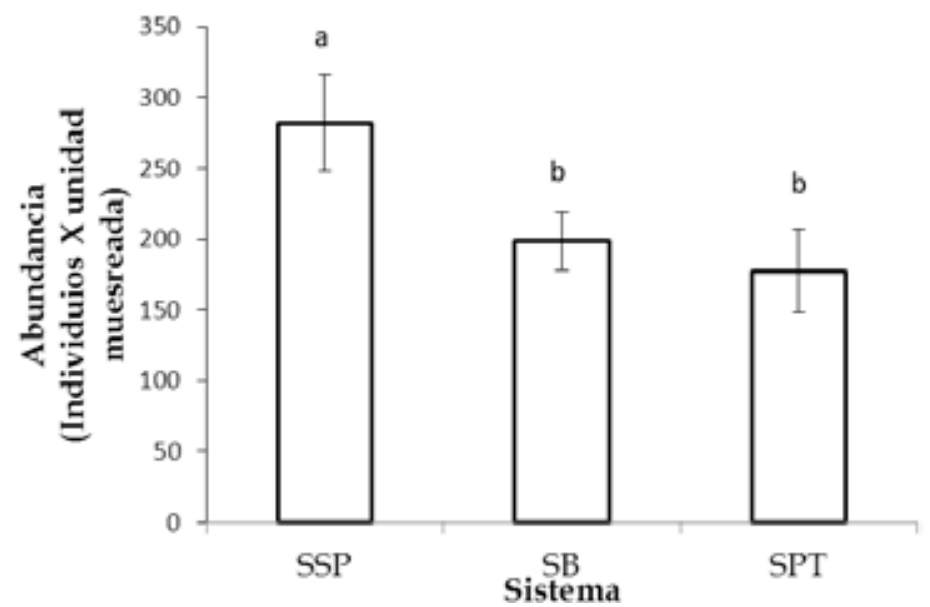

Figura 1. Diferencias en la abundancia de macro fauna en los tres sistemas: sistema silvopastoril (SSP), sistema bosque latifoliado (SB) y sistema de potrero tradicional (SPT) de la micro cuenca Tomabú, Nicaragua. 2012. Letras minúsculas distintas indican diferencias significativas en la comparación de medias $(p \leq 0.05)$ entre sistemas.

Estos resultados son parecidos a los encontrados en potreros y sistemas silvopastoriles colombianos (Gómez y Velásquez, 1999) y también coinciden con los reflejados por Sánchez y Crespo, (2004) en Cuba, en cuanto a la mayor abundancia de macrofauna en los sistemas silvopastoriles respecto a los pastos en monocultivos. En cambio difieren con los presentados por Brown et al., (2001), y Cabrera (2011), donde el bosque tiene una mayor abundancia de individuos que los pastizales.

En cuanto a la diversidad de las poblaciones de macro invertebrados edáficos (Cuadro 2), los valores más altos para el índice de Shannon $\left(\mathrm{H}^{\prime}\right)$ fueron para el sistema bosque y menores para los sistemas silvopastoril y potrero tradicional, lo que coindice con Socarras et al., (2011) en Cuba, donde el pastizal mostró estimados de densidad inferiores a los reportados para los usos bosque pero muy semejantes a los de otros pastizales, mas difiere con los hallazgos de Cerón et al., (2009) en Colombia donde la densidad total de la macrofauna y de los artrópodos fue mayor en Bosque que en otros usos.
Cuadro 2. Valores de la diversidad, Índice de Shannon (valor de $\mathbf{H}^{\prime}$ ), por sistema para la micro cuenca Tomabú, Nicaragua. 2012

\begin{tabular}{llll}
\hline Sistema & SB & SPT & SSP \\
\hline Valor de $\mathrm{H}^{\prime}$ & 1.93 & 1.69 & 1.68 \\
\hline
\end{tabular}

Este resultado comprueba la hipótesis planteada para el estudio que la biodiversidad de macrofauna del suelo es mayor en el bosque que en los sistemas de producción pecuaria (sistema silvopastoril y potrero tradicional).

Sin embargo, no hubo diferencias significativas entre la diversidad de macrofauna de los tres sistemas (Figura 2), lo cual probablemente se deba a que los sistemas en estudio poseen cierto grado de intervención o alteración antropogénica donde pueden colonizar igualmente familias con características semejantes de tolerancia a un variado rango de condiciones edafoclimáticas y, por tanto, adaptables y resistentes a las perturbaciones inducidas. (Cabrera et al., 2011).

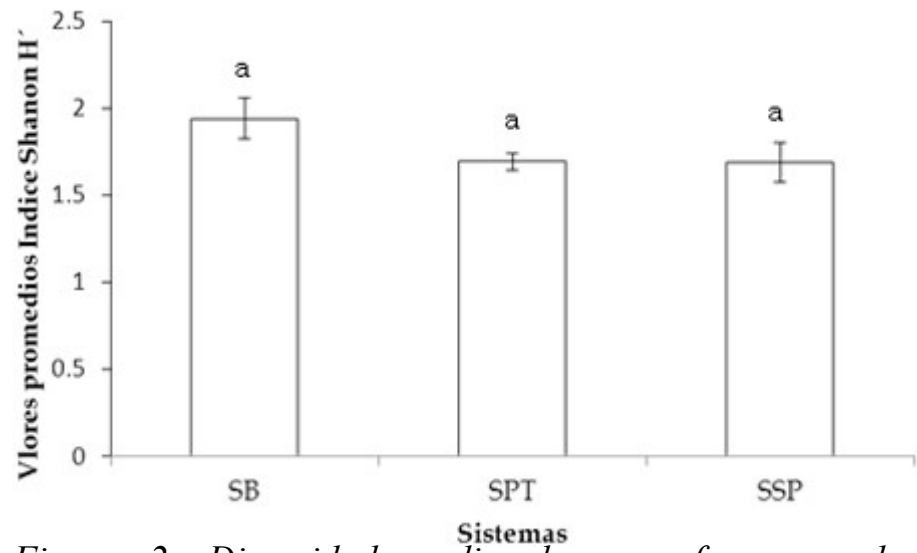

Figura 2. Diversidad media de macrofauna en los tres sistemas, de la micro cuenca Tomabú, Nicaragua. 2012. Letras minúsculas distintas indican diferencias significativas $(p \leq 0,05)$ entre sistemas.

\section{Características físico-químicas del suelo}

En el Cuadro 3 se observa que los tres sistemas considerados en este estudio presentaron valores de $\mathrm{pH}$ medianamente acido, así como un elevado contenido de materia orgánica. La capacidad de intercambio 
catiónico fue alta para los sistemas silvopastoriles (SSP), los sistema de potrero tradicional (SPT) y muy alta para el sistema bosque (SB).

En los análisis de macro elementos los valores de $\mathrm{N}$, $\mathrm{Ca}$ y $\mathrm{Mn}$ fueron altos para los tres sistemas mientras que el $\mathrm{P}$ resultó en niveles bajo, como suele ocurrir en suelos no fertilizados. El valor de $\mathrm{K}$ es bajo en SSP y medio para SB y SPT.

En análisis para micro elementos el valor del Fe fue muy alto para SSP y SPT pero bajo en SB; del Cu fue bajo para SB, medio para SPT y alto para SSP, el Zn fue muy bajo para SPT y bajo para SSP y BL. Esta valoración siguiendo los parámetros de Quintana et al., (1983) del laboratorio de suelos y aguas (UNA, 2012). La estructura de los suelos resultó granular para los SB y granular o laminar en los SPT y SSP. La textura arcillosa predominó en los SSP y SPT y resultó franco arcillosa para SB, lo cual coincide con los datos de INIFOM, (2012).

Cuadro 3. Características físico químicas $\bigcirc$ de los suelos de tres sistemas en estudio: sistema silvopastoril (SSP), sistema bosque (SB) y sistema potrero tradicional (SPT) de la micro cuenca Tomabú (Nicaragua).

\begin{tabular}{lllll}
\hline \multirow{2}{*}{ Caracteristicas } & \multicolumn{3}{c}{ Sistema } \\
\cline { 3 - 5 } & & SSP & SB & SPT \\
\hline$\square \mathrm{pH}$ & & 6.17 & 6.36 & 6.16 \\
$\mathrm{MO}$ & $\%$ & 4.34 & 5.27 & 4.12 \\
$\mathrm{~N}$ & $\%$ & 0.217 & 0.264 & 0.20 \\
$\mathrm{P}$ & $\mathrm{ppm}$ & 6.15 & 6.01 & 6.41 \\
$\mathrm{CIC}$ & Meq 100 g-1 suelo & 38.51 & 47.27 & 36.97 \\
$\mathrm{~K}$ & Meq 100 g-1 suelo & 0.18 & 0.25 & 0.29 \\
$\mathrm{Ca}$ & Meq 100 g-1 suelo & 18.34 & 19.47 & 17.86 \\
$\mathrm{Mg}$ & Meq 100 g-1 suelo & 8.49 & 7.50 & 6.93 \\
$\mathrm{Fe}$ & ppm & 75.56 & 3.92 & 53.44 \\
$\mathrm{Cu}$ & ppm & 2.65 & 0.87 & 1.75 \\
$\mathrm{Zn}$ & ppm & 2.60 & 2.26 & 0.89 \\
$\mathrm{Mn}$ & ppm & 72.23 & 84.13 & 58.60 \\
\hline
\end{tabular}

o Promedio de datos de tres parcelas, para cada sistema muestreado.

Porcentaje de Hidrogeno $(\mathrm{pH})$, materia orgánica (MO), capacidad de intercambio catiónico (CIC), Nitrógeno (N), Fosforo (P), Potasio (K), Calcio (Ca), Magnesio (Mg), Hierro (Fe), Cobre $(\mathrm{Cu})$, Zinc $(\mathrm{Zn})$, Manganeso (Mn).

Debido a la cantidad de datos con los que se contaba para el estudio, los análisis estadísticos no reflejan significancia en las diferencias entre los datos de suelo para los tres sistemas, sin embargo, se pueden ver variaciones numéricas entre los valores de las características química de los suelos en estudio, que se relacionan con sus características físicas, es así que los suelos del sistema bosque, con una textura franco arcilloso y una estructura blocosas tienen valores más altos de $\mathrm{pH}, \mathrm{MO}, \mathrm{CIC}, \mathrm{N}$ y $\mathrm{Mn}$ en comparación con los de los sistemas silvopastoril y potrero tradicional que tienen valores similares entre sí, con textura arcillosa y estructura blocosa y laminar respectivamente.

Los valores numéricos para los macro elementos $\mathrm{P}$, $\mathrm{K}, \mathrm{Mg}$ y los micro elementos $\mathrm{Fe}, \mathrm{Cu}$, sin embargo registran valores más bajos en el sistema bosque que en los otros dos sistemas, esto se puede asociar a que los sistemas de uso pecuario en algún momento ( 5 años atrás) fueron utilizados también con uso agrícola donde recibieron adiciones de fertilizantes químicos que aún persisten en el suelo. Mientras tanto los valores de $\mathrm{Zn}$ resultan menores en el SPT y mayores en el SSP y SB sucesivamente.

En el Cuadro 4 se muestran relaciones lineales entre las características del suelo con la diversidad y abundancia, sin embargo estas tienen mayor relación con la diversidad que con la abundancia y las variables de suelo que mejor relación tienen con la diversidad son el $\mathrm{pH}, \mathrm{P}, \mathrm{Mg}$ y $\mathrm{Mn}$ con mayores valores de R2, en cambio la abundancia tiene mejores relaciones con el $\mathrm{Py} \mathrm{Cu}$. 
Cuadro 4. Resultados de análisis de regresión para las variables de suelo comparadas con la diversidad (D) y abundancia (A). Valor de R2 y P-valor, para los tres sistemas en estudio en la micro cuenca de Tomabú, Nicaragua.

\begin{tabular}{lclll}
\hline Característica del & \multicolumn{2}{c}{$\mathbf{R}^{2}$} & \multicolumn{2}{l}{ P-value } \\
\cline { 2 - 5 } \multicolumn{1}{c}{ suelo } & D & A & D & A \\
\hline $\mathrm{pH}$ & 0.597 & 0.003 & 0.014 & 0.897 \\
$\mathrm{MO}$ & $2 \mathrm{E}-06$ & $1 \mathrm{E}-05$ & 0.007 & 0.98 \\
$\mathrm{~N}$ & $5 \mathrm{E}-04$ & 0.001 & 0.95 & 0.953 \\
$\mathrm{P}$ & 0.381 & 0.279 & 0.76 & 0.279 \\
$\mathrm{CIC}$ & 0.006 & 0.089 & 0.844 & 0.434 \\
$\mathrm{~K}$ & 0.007 & 0.003 & 0.832 & 0.884 \\
$\mathrm{Ca}$ & 0.02 & 0.002 & 0.718 & 0.92 \\
$\mathrm{Mg}$ & 0.214 & 0.094 & 0.21 & 0.422 \\
$\mathrm{Fe}$ & 0.016 & 0.12 & 0.74 & 0.343 \\
$\mathrm{Cu}$ & 0.035 & 0.424 & 0.62 & 0.057 \\
$\mathrm{Zn}$ & 0.085 & 0.057 & 0.447 & 0.534 \\
$\mathrm{Mn}$ & 0.254 & 0.044 & 0.166 & 0.59 \\
\hline
\end{tabular}

Diversos autores manifiestan que el contenido de materia orgánica, el estatus nutricional, el $\mathrm{pH}$ y la textura del suelo pueden determinar la composición y abundancia de las comunidades edáficas. (Lavelle, 1995; Zerbino, 2010; Rendon et al., 2011).

Domínguez et al., 2001 y Cerón et al., 2009, han encontrado que los índices de biodiversidad están relacionados con la materia orgánica que se encuentre en el suelo y que diferencias en la dinámica de la materia orgánica inciden en la macrofauna pero también que $\mathrm{pH}$ más ácido disminuye la densidad de macrofauna, esto coincide con la situación encontrada en la microcuenca Tomabú donde los contenidos de materia orgánica y el $\mathrm{pH}$ tienen valores similares en los tres sistemas en estudio. Esto también explicaría que no se presente diferencias significativas en los valores de diversidad y que los valores de diversidad del bosque sean similares a los sistemas aledaños.

\section{CONCLUSIONES}

En la microcuenca de Tomabú existe una mayor diversidad de macrofauna en el sistema de bosque comparada con los sistemas de producción pecuaria del estudio (sistema silvopastoril y potrero tradicional), sin embargo estas diferencias son no significativas de acuerdo a los análisis estadísticos realizados en este estudio, lo cual se relaciona con las características del suelo de $\mathrm{pH}$ y materia orgánica.

La riqueza taxonómica fue mayor en el sistema silvopastoril que en los sistemas de bosque y de potrero tradicional, ya que se identificaron un mayor número de órdenes e igualmente es en el sistema silvopastoril donde se encuentra la mayor abundancia de individuos para este estudio.

De las variables de suelo estudiadas se concluye que el $\mathrm{pH}$ fue determinante en la diversidad de poblaciones mientras que el cobre y el fósforo incide en la abundancia para los tres sistemas en estudio en la microcuenca Tomabú.

\section{AGRADECIMIENTOS}

A la UNAN - FAREM, BICU, UAB y Fundación Autónoma Solidaria, a los coordinadores de la maestría: Alejandrina Herrera, y Rene Cassell. A CARE-MARENA - PIMCHAS por su colaboración técnica gracias a la cooperación de los Pueblos y Gobiernos de Canadá y Nicaragua mediante un Memorando de Entendimiento firmado entre Canadá y Nicaragua. Al equipo de trabajo de la Estación experimental para el Trópico Seco El Limón UNANFAREM Estelí: Josué Urrutia, Kenny López, Verónica Ruiz, Oscar Rafael. A los señores Roberto Cruz, Alexis Cruz, Vilma Castellón, Oscar Escoto, Santos Tinoco, Ramón Benavidez, Zacarías Mairena y Fabio Mairena, productores y productoras de la micro cuenca Tomabú que brindaron acceso a sus fincas. 


\section{REFERENCIAS BIBLIOGRÁFICAS}

Aloé J, M. M. Toribio. 2007. Análisis de suelos guía práctica de muestreo. Investigación y Desarrollo 12: 1-8. http://www.profertilnutrientes.com.ar/ images/archivos/?id=128\%20 (Mayo 2013).

Altieri M, A. 1999. The ecological role of biodiversity in agro ecosystems. Agriculture Ecosystems Environ. 74:19-31.

Anderson J, M. 1975. The enigma of soil animal species diversity. In: Progress in Soil Zoology. Proc. 5th International Colloquium on Soil Zoology 1973. pp: 51-8.

Arroyo J, C Iturrondobeitia J, I Caballero A, C Gonzales S. 2003. Las comunidades de micro y meso artrópodos de parcelas experimentales de cultivo de secano en un muestreo de invierno. España. Boletin. Asoc. Española de Entom. 27:4145.

Brown G, G, C Fragoso, I Barois, P Rojas, C Patrón J, J Bueno, G Moreno A, P Lavelle, V Ordaz, C Rodríguez. 2001. Diversidad y rol funcional de la macrofauna edáfica en los ecosistemas tropicales mexicanos. Conservación y manejo sostenible de la biodiversidad bajo el suelos. Acta Zoológica Mexicana 1: 79-100

Cabrera G, N Robaina, D Ponce L. 2011. Riqueza y abundancia de la macrofauna edáfica en cuatro usos de la tierra en las provincias de Artemisa y Mayabeque, Cuba. Pastos y Forrajes, 34: 313-330.

Casasola F, Ibrahim M, Harvey C, Kleinn C, 2001. Caracterización y productividad de sistemas silvopastoriles tradicionales en Moropotente, Estelí, Nicaragua. Agroforesterìa en las Américas 8: 17-20.

Castro P, J. H, O Burbano H, C Bonilla C. R. 2007. Abundancia y biomasa de organismos edáficos en tres usos del terreno en el altiplano de pasto, Colombia. Acta Agronómica 56: 127-130.

Cerón P, H García. 2009. Propiedades del suelo en bosque y pajonal; reserva natural Pueblo Viejo, Nariño, Colombia. UDCAActualidad y divulgación científica. 12: 113-120.
Dufrêne M, P Legendre. 1997. Species assemblages and indicator species: the need for a flexible asymmetrical approach. Ecological Monographs 67: 345-366.

Delabie J, H.C, H Fowler G.1990. Cryptic species assemblages in tropical and temperate latitudes. Pp. 695-696. In: Veeresh, G.K., B. Mallik and C.A. Viraktamath (eds). Social Insects and the Environment. Proceedings of the 11th International Congress of IUSSI. Bangalore, India. Oxford \& IBH Publishing Co. PVT. LTD. New Delhi, India. $765 \mathrm{pp}$.

Domínguez G, A, Laborda R, Martínez D F, Roselló O J. 2003; Evaluación de microartrópodos en suelos de cítricos ecológicos y convencionales. Posibilidades de uso como bioindicadores, dentro de "L'Agricultura Ecológica a la Comunitat Valenciana", Actas del III Congreso valenciano de Agricultura Ecológica (Castelló, diciembre 2002); p. 315-330; Ed. Universitat Jaume I http://fci.uib. es/Servicios/libros/conferencias/seae/Evaluacionde-microartropodos-en-suelos-de.cid221700

Feijoo A, E Knapp. 1998. El papel de los macro invertebrados como indicadores de fertilidad y perturbación de suelos de ladera. Suelos Ecuatoriales 28: 254-259.

Ghilarov M, S. 1977. Why so many species and so many individuals can coexist in the soil. In: Soil Organisms as Components of" Ecosystems. EcoL Bull Stockholm 25: 593-7.

Gil M, J.1997. Estudio de los Ácaros Oribátidos de pinares incendiados de un sector de la cara sur de la Sierra de Gredos (Ávila). Tesis doctoral Universidad Complutense de Madrid. 433 p.

Gómez J, E, Velásquez J E. 1999. Proceso integral de recuperación y manejo de praderas, condición fundamental para el desarrollo ganadero en Caquetá. Boletín Técnico Corpoica-Pronatta. 42 p. González V, N. S. Ochoa G, C Pozo, B G Ferguson, L JRangel R, S L Arriaga W, A Ponce, C Kampichler. 2011. Un paisaje neotropical: perspectiva multitaxonómica. Biolog. Tropic. 59: 1433-1451. 
Huerta E, Rodríguez J, Castillo I, Cruz M. E, García R. 2008. Relación entre la fertilidad del suelo y su población de macro invertebrados. TERRA Latinoamericana, 26: 171-181.

INIFOM (Instituto Nicaragüense de Fomento Municipal) 2012. Ficha Municipal de La Trinidad. Estelí, Nicaragua.

http://www.inifom.gob.ni/municipios/documentos/ ESTELI/la_trinidad.pdf

Lavelle P, Kohlman B. 1984. Estude quantitative de la macrofaune do sol dans une forest tropicale. Pedobiologia, 24: 834 - 845.

Lavelle P. 1995. Faunal activities and soil processes: adaptative strategies that determine ecosystem function ISSS Congress, 15th, Acapulco, México.

Magurran A, E. 1988. Ecological diversity and its measurement. Princeton University Press, New Jersey, 179 pp.

Mahecha L, 2002. El silvopastoreo: una alternativa de producción que disminuye el impacto ambiental de la ganadería bovina. Ciencias Pecuarias 15:213

MARENA (Ministerio del Ambiente y los Recursos Naturales) CARE (Cooperativa de remesas al exterior) PIMCHAS (Programa integrado de manejo de cuencas hidrográficas agua y saneamiento). 2008. Caracterización biofísica y socioeconómica de la parte media de la subcuenca del rio Viejo. Estelí, Nicaragua.

MARENA / INAFOR (Instituto Nacional Forestal). 2002. Guía de Especies Forestales de Nicaragua. Orgut Consulting AB 1 ra Ed. Managua, Nicaragua, Editora de Arte. 304 p. ISBN 99924-34-17-1

http://www.inafor.gob.ni/images/documentos/ Guia\%20de\%20especies\%20forestales\%20en\%20 nicaragua/Gu $\% \mathrm{C} 3 \% \mathrm{ADa} \% 20 \mathrm{de} \% 20$ Especies $\% 20$ Forestales.pdf

Moreira M, S Fatima, Huisingy E Jeroen, Bignell H David. 2012. Manual de Biología de Suelos Tropicales. Muestreo y Caracterización de la biodiversidad bajo suelo. Capítulo 4:Collembola, acari y otra mesofauna del suelo: el método Berlese. México 149-162.

Peredo F, Santiago C, Barrera P, E. Parada, M Vega.
2012. Análisis temporal de la taxocenosis y biocenosis de la mesofauna edáfica en plantaciones de vaccinium sp. con manejo orgánico en el centrosur de Chile. Agrociencia 46: 163-173.

Piura J, L. 1994. Introducción a la metodología de la investigación científica. 1ra ed. Publicación científica de la Escuela de Salud Pública de Nicaragua.114p

Rendón S, P, F Artunduaga L, R Ramírez P, J. A. Quiroz G, E. I. Leiva R. 2011. Los macro invertebrados como indicadores de la calidad del suelo en cultivos de Mora, pasto y Aguacate. Colombia. Facultad Nacional Agraria de Medellín 64:5793-5802.

Rodríguez T, I. V. 2012. Identificación de ácaros que afectan cultivos de naranja valencia (Citrus Sinensis L.) en el núcleo sur occidental de Colombia y establecimiento de dinámica de población y fenología de algunas especies de importancia económica. Tesis doctoral. Universidad Nacional de Colombia, sede Palmira. 175 p.

Socarrás A, N Robaina. 2011. Caracterización de la mesofauna edáfica bajo diferentes usos de la tierra en suelo ferralítico rojo de Mayabeque y Artemisa. Pastos y Forrajes, 34: 185-198.

Sánchez S, G Crespo. 2004. Comportamiento de la macrofauna del suelo en pastizales con gramíneas puras o intercaladas con leucaena. Pastos y Forrajes. 27: 347-353.

Stanton N, L. 1979. Patterns of species diversity in temperate and tropical litter mites. Ecology 60: 295-304.

UNA (Universidad Nacional Agraria). 2012. Instrucciones para el levantamiento de muestras. Laboratorio de suelos y aguas. Managua Nicaragua. Zerbino B, S. M. 2010. Evaluación de la macrofauna del suelo en rotaciones cultivos-pasturas con laboreo convencional. Acta Zoológica Mexicana Número Especial 2: 189-202.

Zerbino B, N Altier, A Morón, C Rodríguez. 2008. Evaluación de la macrofauna del suelo en sistemas de producción en siembra directa y con pastoreo. Agrociencia 12: 44-55. 